\title{
Partial stochastic asymptotic stability of neutral stochastic functional differential equations with Markovian switching by boundary condition
}

\author{
Dezhi Liu ${ }^{1,2 *}$, Weiqun Wang ${ }^{1}$, Oleksiy Ignatyev ${ }^{3}$ and Wei Zhang ${ }^{2}$
}

${ }^{*}$ Correspondence:

mathliudz@yahoo.com.cn

${ }^{1}$ Department of Applied

Mathematics, Nanjing University of

Science and Technology, Nanjing

Jiangsu 210094, China

${ }^{2}$ School of Statistics and Applied

Mathematics, Anhui University of

Finance and Economics, Bengbu,

Anhui 233030, China

Full list of author information is

available at the end of the article

\begin{abstract}
The partial stochastic asymptotic stability of neutral stochastic functional differential equations with Markovian switching (NSFDEwMSs) has been investigated, the sufficient conditions for partial stochastic asymptotic stability have been given. We have used the boundary condition and $L V \leq 0$ to replace the condition $L V<0$ and have generalized some results of Sharov and Ignatyev to cover a class of much more general NSFDEwMSs.

MSC: $39 \mathrm{~A} 11 ; 60 \mathrm{H} 10 ; 37 \mathrm{H} 10$
\end{abstract}

Keywords: stochastic asymptotic stability; partial; Markov chain; neutral; functional differential equation

\section{Introduction}

In recent years, Lyapunov's second method has been a very important method to study the stability of a stochastic differential equation, which has attracted much attention since it is not only academically challenging, but also of practical importance, and has played an important role in many fields such as insurance, finance, population dynamic, control (see, e.g., Øsendal [1]). Recently, much work has been done on stochastic differential equations with Markovian switching (see, e.g., Mao and Yuan [2]). In particular, significant work has appeared on the stability of stochastic differential delay equations with Markovian switching (see, e.g., Kolmanovskii et al. [3], Luo [4], Luo et al. [5], Mao et al. [6], Yuan and Mao [7], Yuan et al. [8], Mao et al. [9], Liu [10, 11]).

On the other hand, many dynamical systems depend not only on present and past states, but also involve derivatives with delays. Motivated by chemical engineering systems as well as the theory of aero elasticity, many researchers have been interested in the neutral stochastic functional differential equations, and the neutral part, which has been used in investigating the qualitative properties of derivatives of delays for dynamical systems, has been added to all kinds of equations. In particular, the significant work has been done (see, e.g., Mao [12], Luo et al. [13]).

The global stability of SDEwMSs with respect to sample paths or moments, which is too strong for many cases, appeared in many more papers. So, the notion of the partial stability (see, e.g., Peiffer and Rouche [14], Rouche et al. [15]) has been involved, and the second

(c) 2012 Liu et al.: licensee Springer. This is an Open Access article distributed under the terms of the Creative Commons Attribution License (http://creativecommons.org/licenses/by/2.0), which permits unrestricted use, distribution, and reproduction in any medium, provided the original work is properly cited. 
method of Lyapunov as an indispensable tool has been used to investigate the partial stability (see, e.g., Sontag and Wang [16], Vorotnikov [17], Vorotnikov and Rumyantsev [18], Ignatyev [19, 20]).

However, in the process of investigating the qualitative properties of equilibria and boundedness properties of motions of dynamical systems, the partial stability as a new concept plays the key role, and the systems are often determined by all kinds of equations, including stochastic differential equations, of course. Motivated by the previously mentioned topic, and since the results of the partial stochastic asymptotic stability of neutral stochastic functional differential equations with Markovian switching are very few, we make the attempt to study this topic to fill the gap.

This paper is organized as follows. In Section 2, we present some basic preliminaries and the form of neutral stochastic functional differential equations with Markovian switching. In Section 3, the sufficient conditions for partial stochastic asymptotic stability have been obtained and proofs have been given. In Section 4, some well-known results are generalized in the remarks. In Section 5, there is the discussion about the main results.

\section{Preliminaries and definitions}

Let $\left\{\Omega, \mathcal{F},\{\mathcal{F}\}_{t \geq 0}, \mathbf{P}\right\}$ be a complete probability space with a filtration satisfying the usual conditions, i.e., the filtration is continuous on the right and $\mathcal{F}_{0}$ contains all $P$-zero sets. $B(t)$ is an $m$-dimensional Brownian motion defined on the probability space. Let $C\left([-\tau, 0] ; R^{n}\right)$ denote the family of functions $\varphi$ from $[-\tau, 0]$ to $R^{n}$ that are right-continuous and have limits on the left. $C\left([-\tau, 0] ; R^{n}\right)$ is equipped with the norm $\|\varphi\|=\sup _{-\tau \leq s \leq 0}|\varphi(s)|$ and $|x|=\sqrt{x^{T} x}$ for any $x \in R^{n}$. If $A$ is a vector or matrix, its trace norm is denoted by $|A|=\sqrt{\operatorname{trace}\left(A^{T} A\right)}$, while its operator norm is denoted by $\|A\|=\sup \{|A x|:|x|=1\}$. Denote $C_{\mathcal{F}_{0}}^{b}\left([-\tau, 0] ; R^{n}\right)$ to be the family of all $\mathcal{F}_{0}$-measurable bounded $C\left([-\tau, 0] ; R^{n}\right)$-valued random variables $\xi=\{\xi(\theta):-\tau \leq \theta \leq 0\}$. Let $p>0, t \geq 0, L_{\mathcal{F}_{t}}^{p}\left([-\tau, 0] ; R^{n}\right)$ denote the family of all $\mathcal{F}_{t}$ measurable, $C\left([-\tau, 0] ; R^{n}\right)$-valued random variables $\varphi=\{\varphi(\theta):-\tau \leq \theta \leq 0\}$ such that $\sup _{-\tau \leq \theta \leq 0} E|\varphi(\theta)|^{p}<\infty$.

Let $\left\{r(t), t \in R_{+}=[0,+\infty)\right\}$ be a right-continuous Markov chain on the probability space $\left\{\Omega, \mathcal{F},\{\mathcal{F}\}_{t \geq 0}, \mathbf{P}\right\}$ taking values in a finite state space $S=\{1,2, \ldots, N\}$ with a generator $\Gamma=$ $\left(\gamma_{i j}\right)_{N \times N}$ given by

$$
P(r(t+\Delta)=j \mid r(t)=i)= \begin{cases}\gamma_{i j} \Delta+o(\Delta), & \text { if } i \neq j, \\ 1+\gamma_{i i} \Delta+o(\Delta), & \text { if } i=j,\end{cases}
$$

where $\Delta>0$. Here $\gamma_{i j} \geq 0$ is the transition rate from $i$ to $j$, if $i \neq j$, while

$$
\gamma_{i i}=-\sum_{j \neq i} \gamma_{i j}
$$

We assume that the Markov chain $r(\cdot)$ is independent of the Brownian motion $B(\cdot)$. It is known that almost every sample path of $r(t)$ is a right-continuous step function with a finite number of simple jumps in any finite sub-interval of $R_{+}$.

Consider the following neutral stochastic functional differential equation with Markovian switching:

$$
d\left[x(t)-G\left(x_{t}\right)\right]=f\left(t, x_{t}, r(t)\right) d t+g\left(t, x_{t}, r(t)\right) d B(t), \quad t>0,
$$


with the initial condition $x_{0}=\xi=\left(\xi_{1}, \xi_{2}\right)^{T} \in C_{\mathcal{F}_{0}}^{b}\left([-\tau, 0] ; R^{n}\right)$, where $\xi_{1} \in R^{k}$ and $\xi_{2} \in R^{p}$, $k+p=n$, which is independent of $B(\cdot)$, and $x_{t}=\{x(t+\theta):-\tau \leq \theta \leq 0\}$. Let $\widetilde{x(t)}=x(t)-$ $G\left(x_{t}\right)$. Here, we furthermore assume that

$$
\begin{aligned}
& f: R_{+} \times C\left([-\tau, 0] ; R^{n}\right) \times S \rightarrow R^{n}, \quad g: R_{+} \times C\left([-\tau, 0] ; R^{n}\right) \times S \rightarrow R^{n \times m}, \\
& G: C\left([-\tau, 0] ; R^{n}\right) \rightarrow R^{n} .
\end{aligned}
$$

For our purpose, we impose the following assumptions which can ensure the existence and uniqueness of a solution, denoted by $x(t)$ on $t>0$, for equation (2.1). Let $f$ and $g$ satisfy the local Lipschitz and linear growth condition. That is, for each $d>0$, there exist constants $h>0, C_{d}>0$ and $\kappa \in(0,1)$ such that

$$
\begin{aligned}
& \left|f\left(t, \varphi_{1}, i\right)-f\left(t, \varphi_{2}, i\right)\right|^{2} \vee\left|g\left(t, \varphi_{1}, i\right)-g\left(t, \varphi_{2}, i\right)\right|^{2} \leq C_{d}\left\|\varphi_{1}-\varphi_{2}\right\|^{2}, \\
& |f(t, \varphi, i)|^{2} \vee|g(t, \varphi, i)|^{2} \leq h\left(1+\|\varphi\|^{2}\right), \\
& |G(\varphi)| \leq \kappa\|\varphi\| .
\end{aligned}
$$

Denote $x=\left(x_{1}, x_{2}\right)^{T} \in R^{n}$, where $x_{1} \in R^{k}$ and $x_{2} \in R^{p}, k+p=n$, and the definitions of $\widetilde{x}=\left(\widetilde{x_{1}}, \widetilde{x_{2}}\right)^{T} \in R^{n}$ and $G\left(x_{t}\right)=\left(G_{1}\left(x_{t}\right), G_{2}\left(x_{t}\right)\right)^{T} \in R^{n}$ are similar to $x=\left(x_{1}, x_{2}\right)^{T}$. The domain $B_{K}=\left\{x \in R^{n}:\left|x_{1}\right|<K,\left|x_{2}\right|<\infty\right\}$, and the stopping time $\sigma_{B}$ is the first exit time from the $B_{K}$ of the sample path of the process $x(t)$. Let $K \rightarrow \infty$, then $\sigma_{B} \rightarrow \infty$. Denote the set of functions $\mathcal{C}:=\left\{\phi: R^{+} \rightarrow R^{+}\right.$, continuous, monotonically increasing and $\left.\phi(0)=0\right\}$.

Denote by $C^{1,2}\left(R_{+} \times R^{n} \times S ; R_{+}\right)$the family of all non-negative functions $V(t, x, i)$ on $R_{+} \times$ $R^{n} \times S$, which are twice continuously differential with respect to $x$ and once continuously differential with respect to $t$. For any $(t, x, i) \in R_{+} \times R^{n} \times S$, define an operator $L V$ by

$$
\begin{aligned}
L V\left(t, x_{t}, i\right)= & V_{t}(t, \widetilde{x(t)}, i)+V_{x}(t, \widetilde{x(t)}, i) f\left(t, x_{t}, i\right) \\
& +\frac{1}{2} \operatorname{trace}\left[g^{T}\left(t, x_{t}, i\right) V_{x x}(t, \widetilde{x(t)}, i) g\left(t, x_{t}, i\right)\right] \\
& \left.+\sum_{j=1}^{N} \gamma_{i j} V(t, \widetilde{x(t}), j\right),
\end{aligned}
$$

where

$$
V_{t}=\frac{\partial V(t, x, i)}{\partial t}, \quad V_{x}=\left(\frac{\partial V(t, x, i)}{\partial x_{1}}, \ldots, \frac{\partial V(t, x, i)}{\partial x_{n}}\right), \quad V_{x x}=\left(\frac{\partial^{2} V(t, x, i)}{\partial x_{i} \partial x_{j}}\right)_{n \times n} .
$$

Definition 2.1 The trivial solution $x(t)$ of equation (2.1) is said to be partial stochastic stability with respect to $x_{1}(t)$ if, for any $\epsilon>0$ and $\epsilon_{1}>0$, there exists a $\delta=\delta\left(\epsilon, \epsilon_{1}\right)>0$ such that

$$
P\left\{\sup _{t \geq 0}\left|x_{1}(t)\right|>\epsilon\right\}<\epsilon_{1},
$$

whenever $\|\xi\|<\delta$. 
Definition 2.2 The trivial solution $x(t)$ of equation (2.1) is said to be partial stochastic asymptotic stability with respect to $x_{1}(t)$ if it is the $x_{1}(t)$-stochastic stability and for any $\epsilon \in(0,1)$, there exists a $\delta_{0}=\delta_{0}(\epsilon)$ such that

$$
P\left\{\lim _{t \rightarrow \infty}\left|x_{1}(t)\right|=0\right\} \geq 1-\epsilon,
$$

whenever $\|\xi\|<\delta_{0}$.

\section{Main results}

First of all, we should present Sharov's theorem [21].

Theorem 3.1 (Sharov [21]) If in the domain $B_{K} \times R_{+}$there exists a function $V(x, t)$ which satisfies the following conditions:

(I) $\alpha\left(\left|x_{1}\right|\right) \leq V(t, x) \leq \beta\left(\left|x_{1}\right|\right)$, where $\alpha, \beta \in \mathcal{C}$;

(II) $L V \leq-\gamma\left(\left|x_{1}\right|\right)$, where $\gamma \in \mathcal{C}$;

then the trivial solution $x(t) \equiv 0$ of system (2.1) is $x_{1}(t)$-stochastic asymptotic stability.

The condition of Theorem 3.1 is more difficult to be satisfied, which is demonstrated by the following example.

Example 3.1 Consider the system of stochastic differential equations

$$
\begin{aligned}
& d x_{1}=-a x_{1}^{3} x_{2}^{2} d t+\sqrt{a} x_{1}^{2} x_{2} d B_{1}(t), \\
& d x_{2}=b x_{1} d t+c x_{2} d B_{2}(t)
\end{aligned}
$$

where $a, b, c$ are constants and $a b c \neq 0$. The system has the trivial solution $x_{1}=0, x_{2}=0$.

Denote $V:=x_{1}^{2}$, we have $L V=-2 a x_{1}^{4} x_{2}^{2}+2 a x_{1}^{4} x_{2}^{2}=0$.

Now, by Theorem 3.1, we cannot give whether the solution of (3.1) is stochastic asymptotic stability or not. So, we present the main results of this paper as follows.

Theorem 3.2 Let there exist a non-negative functional $V(t, x, i) \in C^{1,2}\left(R_{+} \times B_{K} \times S ; R_{+}\right)$ such that

(I) $\mu_{1}\left(\left|x_{1}\right|\right) \leq V(t, x, i) \leq \mu_{2}\left(\left|x_{1}\right|\right)$, where $\mu_{1}, \mu_{2} \in \mathcal{C}$;

(II) $L V \leq 0$;

(III) (Boundary condition) For any sufficiently small $\eta>0, \theta>0$, any solution $x(t)$ of equation (2.1), beginning in the domain $\left\{\eta<\left|x_{1}(t)\right|<\theta\right\}$, is such that

$$
\rho=\inf \left\{t: x_{1}(t)=\eta\right\}<\infty, \text { a.s. }
$$

Then the trivial solution of equation (2.1) is said to be partial stochastic asymptotic stability with respect to $x_{1}(t)$.

Before giving the proof of Theorem 3.1, we should present the following lemma.

Lemma 3.1 Let $V(t, x, i)$ be a function in the class $C^{1,2}\left(R_{+} \times B_{K} \times S ; R_{+}\right)$, bounded in the domain $\left(R_{+} \times B_{K} \times S\right)$, and suppose that $L V \leq 0$ in this domain. Then the process 
$\left.V\left(\sigma_{B} \wedge t, \widetilde{x\left(\sigma_{B} \wedge t\right.}\right), r\left(\sigma_{B} \wedge t\right)\right)$ is a supermartingale so that $E V\left(\sigma_{B} \wedge t, \widehat{x\left(\sigma_{B} \wedge t\right)}, r\left(\sigma_{B} \wedge t\right)\right) \leq$ $V\left(0, x_{0}, i_{0}\right)$, for $x(t) \in B_{K}$.

Proof As the generalized Itô formula, we have

$$
V\left(\sigma_{B} \wedge t, \widetilde{x\left(\widetilde{\sigma_{B} \wedge t}\right)}, r\left(\sigma_{B} \wedge t\right)\right)=V\left(0, x_{0}, i_{0}\right)+\int_{0}^{\sigma_{B} \wedge t} L V\left(s, x_{s}, r(s)\right) d s+M\left(\sigma_{B} \wedge t\right)
$$

where $M\left(\sigma_{B} \wedge t\right)$ is the martingale.

For any $s_{1}, 0 \leq s_{1} \leq \sigma_{B} \wedge t$ and $L V \leq 0$, we derive that

$$
\begin{aligned}
E V & \left(\sigma_{B} \wedge t, \widehat{x\left(\sigma_{B} \wedge t\right)}, r\left(\sigma_{B} \wedge t\right) \mid \mathcal{F}_{s_{1}}\right) \\
& =V\left(0, x_{0}, i_{0}\right)+E\left(\int_{0}^{\sigma_{B} \wedge t} L V\left(s, x_{s}, r(s)\right) d s \mid \mathcal{F}_{s_{1}}\right)+E\left(M\left(\sigma_{B} \wedge t\right) \mid \mathcal{F}_{s_{1}}\right) \\
& \leq V\left(0, x_{0}, i_{0}\right)+E\left(\int_{0}^{s_{1}} L V\left(s, x_{s}, r(s)\right) d s \mid \mathcal{F}_{s_{1}}\right)+M\left(s_{1}\right) \\
& =V\left(0, x_{0}, i_{0}\right)+\int_{0}^{s_{1}} L V\left(s, x_{s}, r(s)\right) d s+M\left(s_{1}\right) \\
& =V\left(s_{1}, \widetilde{x\left(s_{1}\right)}, r\left(s_{1}\right)\right) .
\end{aligned}
$$

So, $\left.V\left(\sigma_{B} \wedge t, \widehat{x\left(\sigma_{B} \wedge t\right.}\right), r\left(\sigma_{B} \wedge t\right)\right)$ is a supermartingale. Moreover, due to $L V \leq 0$ in the domain,

$$
\begin{aligned}
& \left.E V\left(\sigma_{B} \wedge t, \widehat{x\left(\sigma_{B} \wedge t\right.}\right), r\left(\sigma_{B} \wedge t\right)\right) \\
& \quad=V\left(0, x_{0}, i_{0}\right)+E\left(\int_{0}^{\sigma_{B} \wedge t} L V\left(s, x_{s}, r(s)\right) d s\right)+E\left(M\left(\sigma_{B} \wedge t\right)\right) \\
& \quad \leq V\left(0, x_{0}, i_{0}\right),
\end{aligned}
$$

so the proof is completed.

Proof of Theorem 3.2 By Lemma 3.1 and Doob's supermartingale convergence theorem, the process $V\left(\sigma_{B} \wedge t, x\left(\sigma_{B} \wedge t\right), r(t)\right)$ is a supermartingale, and we can get

$$
\left.\lim _{t \rightarrow \infty} V\left(\sigma_{B} \wedge t, \widehat{x\left(\sigma_{B} \wedge t\right.}\right), r(t)\right)=c, \quad \text { a.s. }
$$

Denote $\Omega_{1}=\left\{\omega: \sigma_{B}=\infty\right\}$. Owing to $L V \leq 0$ and the partial stochastic stability theorem (Sharov [21]), the solution of equation (2.1) is the partial stochastic stability with respect to $x_{1}(t)$, so we have

$$
\lim _{\xi \rightarrow 0} P\left(\Omega_{1}\right)=1
$$

Combining this with condition (III) and for all the paths of $\Omega_{1}$, then

$$
\inf _{t>0}\left|x_{1}(t)\right|=0 .
$$


By the lemma of Has'minskii [22], that is, the coefficients of equation (2.1) satisfy the local Lipschitz and linear growth condition in every domain bounded, and the process $x(t)$ is regular, then the set $\left\{\omega: x_{1}(t, \omega)=0\right\}$ is inaccessible to any sample path of the process if $\xi_{1} \neq 0$, where $\xi=\left(\xi_{1}, \xi_{2}\right)^{T}$, so we get

$$
\liminf _{t \rightarrow \infty}\left|x_{1}(t)\right|=0
$$

Noting the similar definition of $\widetilde{x(t)}=x(t)-G\left(x_{t}\right)$, obviously,

$$
-\left|\widetilde{x_{1}(t)}\right| \geq-\left(\left|x_{1}(t)\right|+\left|G_{1}\left(x_{t}\right)\right|\right) \geq-\left(\left|x_{1}(t)\right|+\kappa\left\|x_{t 1}\right\|\right)
$$

and we get that

$$
\sup _{-\tau \leq t<\infty}\left(-\left|\widetilde{x_{1}(t)}\right|\right) \geq \sup _{-\tau \leq t<\infty}\left[-(1+\kappa)\left|x_{1}(t)\right|\right]
$$

Therefore,

$$
\inf _{-\tau \leq t<\infty}\left|\widetilde{x_{1}(t)}\right| \leq \inf _{-\tau \leq t<\infty}(1+\kappa)\left|x_{1}(t)\right|
$$

and (3.4), we have

$$
\inf _{t>0}\left|\widetilde{x_{1}(t)}\right|=0
$$

Similar to the proof of (3.5), we get

$$
\liminf _{t \rightarrow \infty}\left|\widetilde{x_{1}(t)}\right|=0
$$

Combining this with condition (I) $\left(V(t, x, i) \leq \mu_{2}\left(\left|x_{1}\right|\right)\right)$ and (3.7), we obtain

$$
\liminf _{t \rightarrow \infty} V(t, \widetilde{x(t)}, r(t))=0
$$

Owing to (3.2), the limit

$$
\left.\lim _{t \rightarrow \infty} V\left(\sigma_{B} \wedge t, \widetilde{x\left(\sigma_{B} \wedge t\right.}\right), r(t)\right)=\lim _{t \rightarrow \infty} V(t, \widetilde{x(t)}, r(t))=c
$$

exists for almost all paths in $\Omega_{1}$. By (3.8) and (3.9), we have the limit of the above equation $c=0$, a.s., since the condition (I) $\left(\mu_{1}\left(\left|x_{1}\right|\right) \leq V(t, x, i)\right)$. For all the paths of $\Omega_{1}$, we can imply that

$$
\lim _{t \rightarrow \infty}\left|\widetilde{x_{1}(t)}\right|=0
$$

Moreover,

$$
\limsup _{t \rightarrow \infty}\left|\widetilde{x_{1}(t)}\right|=0
$$


Noting the similar definition of $\widetilde{x(t)}=x(t)-G\left(x_{t}\right)$, obviously,

$$
\left|x_{1}(t)\right| \leq\left|\widetilde{x_{1}(t)}\right|+\left|G_{1}\left(x_{t}\right)\right| \leq\left|\widetilde{x_{1}(t)}\right|+\kappa\left\|x_{t 1}\right\|,
$$

we get that

$$
\sup _{-\tau \leq t<\infty}(1-\kappa)\left|x_{1}(t)\right| \leq \sup _{-\tau \leq t<\infty}\left|\widetilde{x_{1}(t)}\right|
$$

By (3.11), then

$$
\limsup _{t \rightarrow \infty}\left|x_{1}(t)\right|=0
$$

Combining this with conditions (3.5) and (3.12) yields

$$
\lim _{t \rightarrow \infty}\left|x_{1}(t)\right|=0
$$

So, we have obtained that the trivial solution of equation (2.1) is partial stochastic asymptotic stability with respect to $x_{1}$ with (3.3) and (3.13).

\section{Remarks}

Remark 4.1 When $G\left(x_{t}\right) \equiv 0, \tau \equiv 0$ and $r(t) \equiv 0$, equation (2.1) reduces to

$$
d x(t)=f(t, x(t)) d s+g(t, x(t)) d B(t), \quad t>0,
$$

which has recently been studied in Ignatyev [23], that is to say, Theorem 3.1 of Ignatyev [23] has been generalized.

Remark 4.2 The operator $L V$ of equation (4.1) of Theorem 3.1 (Sharov [21]) must be negative, but we can obtain the partial stochastic asymptotic stability of equation (2.1) under $L V$ being non-positive $(L V \leq 0)$, which makes the conditions of the solution more feasible to be satisfied.

\section{Discussion of main results}

Lyapunov methods have been developed to research the conditions of the partial asymptotic stochastic stability of neutral stochastic functional differential equations with Markovian switching. We have the non-positive Lyapunov operator and boundary condition to weaken the conditions of the previous theorems, but there is a small problem that the boundary condition of Theorem 3.2 is a stochastic domain. We will obtain the equivalent and practical results for the boundary condition in the future investigation.

Competing interests

The authors declare that they have no competing interests.

Authors' contributions

The authors have achieved equal contributions to each part of this paper. All the authors read and approved the final manuscript. 


\section{Author details}

'Department of Applied Mathematics, Nanjing University of Science and Technology, Nanjing, Jiangsu 210094, China.

${ }^{2}$ School of Statistics and Applied Mathematics, Anhui University of Finance and Economics, Bengbu, Anhui 233030, China.

${ }^{3}$ Department of Statistics and Probability, Michigan State University, A408 Wells Hall, East Lansing, MI 48824-1027, USA.

\section{Acknowledgements}

The authors are very grateful to the anonymous referees for their valuable suggestions. This work was partially supported by HSSF for the Ministry of Education (10YJC630143), NNSF of China (61074006, 71171001), NSSF of China (12BTJ008), PNSF of Anhui (1208085OG131, KJ2011B005, 2011SORL068).

Received: 25 August 2012 Accepted: 3 December 2012 Published: 19 December 2012

\section{References}

1. Øsendal, B: Stochastic Differential Equations, 5th edn. Springer, Berlin (2003)

2. Mao, X, Yuan, C: Stochastic Differential Equations with Markovian Switching. Imperial College Press, London (2006)

3. Kolmanovskii, V, Koroleva, N, Maizenberg, T, Mao, X, Matasov, A: Neutral stochastic differential delay equations with Markovian switching. Stoch. Anal. Appl. 21, 819-847 (2003)

4. Luo, J: Comparison principle and stability of Itô stochastic differential delay equations with Poisson jump and Markovian switching. Nonlinear Anal. 64, 253-262 (2006)

5. Luo, J: Second-order quasilinear oscillation with impulses. Comput. Math. Appl. 46, 279-291 (2003)

6. Mao, X: The Lasalle-type theorems for stochastic differential equations. Nonlinear stud. 7, 307-328 (2000)

7. Yuan, C, Mao, X: Robust stability and controllability of stochastic differential delay equations with Markovian switching. Automatica 40, 343-354 (2004)

8. Yuan, C: Stability in terms of two measures for stochastic differential equations. Dyn. Contin. Discrete Impuls. Syst. 10, 895-910 (2003)

9. Mao, X: Stabilisation of hybrid stochastic differential equations by delay feedback control. Syst. Control Lett. 57, 927-935 (2008)

10. Liu, D, Yang, Y: Doubly perturbed neutral diffusion processes with Markovian switching and Poisson jumps. Appl. Math. Lett. 23, 1141-1146 (2010)

11. Liu, D, Yang, G, Zhang, W: The stability of neutral stochastic delay differential equations with Poisson jumps by fixed points. J. Comput. Appl. Math. 10, 3115-3120 (2011)

12. Mao, X: Stochastic Differential Equations and Their Applications. Horwood, Chichester (1997)

13. Luo, Q, Mao, X, Shen, Y: New criteria on exponential stability of neutral stochastic differential equations. Syst. Control Lett. 55, 826-834 (2006)

14. Peiffer, K, Rouche, N: Liapunov's second method applied to partial stability. J. Méc. 6(2), 20-29 (1969)

15. Rouche, N, Habets, P, Laloy, M: Stability Theory by Liapunov's Direct Method. Springer-Verlag, New York (1977)

16. Sontag, ED, Wang, Y: Lyapunov characterizations of input to output stability. SIAM J. Control Optim. 39(1), 226-249 (2001)

17. Vorotnikov, VI: Partial Stability and Control. Birkhauser, Boston (1998)

18. Vorotnikov, VI, Rumyantsev, V: Stability and Control with Respect to a Part of the Phase Coordinates of Dynamic Systems: Theory, Methods, and Applications. Scientific World, Moscow (2001) (in Russian)

19. Ignatyev, AO: On the partial equiasymptotic stability in functional differential equations. J. Math. Anal. Appl. 268, 615-628 (2002)

20. Ignatyev, OA: On the partial asymptotic stability in nonautonomous differential equations. Differ. Integral Equ. 19(7), 831-839 (2006)

21. Sharov, VF: Partial stability and stabilization of stochastic systems. Avtom. Telemeh. 11, 63-71 (1978) (in Russian)

22. Has'minskii, RZ: Stochastic Stability of Differential Equations. Sijthoff \& Noordhoff, Rockville (1980)

23. Ignatyev, OA: Partial asymptotic stability in probability of stochastic differential equations. Stat. Probab. Lett. 79, 597-601 (2009)

doi:10.1186/1687-1847-2012-220

Cite this article as: Liu et al.: Partial stochastic asymptotic stability of neutral stochastic functional differential equations with Markovian switching by boundary condition. Advances in Difference Equations 2012 2012:220.

\section{Submit your manuscript to a SpringerOpen ${ }^{\circ}$ journal and benefit from:}

- Convenient online submission

Rigorous peer review

- Immediate publication on acceptance

- Open access: articles freely available online

- High visibility within the field

- Retaining the copyright to your article 